\title{
Bearing Fault Diagnosis Using Orthogonal Matching Pursuit with Pulse Atoms Based on Vibration Model
}

\author{
Huijie ZHU \\ College of Field Engineering, PLA University of Science and Technology, Nanjing 210007, China \\ E-mail: zhuhuijiehao@163.com \\ Xinqing WANG \\ College of Field Engineering, PLA University of Science and Technology, Nanjing 210007, China \\ Yanfeng LI \\ College of Field Engineering, PLA University of Science and Technology, Nanjing 210007, China \\ Mengxi LIU \\ Taicang State Taxation, Suzhou 215400, China \\ Tianshuai LIU \\ College of Field Engineering, PLA University of Science and Technology, Nanjing 210007, China
}

\begin{abstract}
In this paper, a new approach to rolling bearing diagnosis is proposed, which applied orthogonal matching pursuit with pulse atoms. Solving orthogonal matching pursuit with pulse atoms (OMP_PA) is an NP-hard problem. With the help of multi-population genetic algorithm, better solution is obtained, and the shortcoming of sensitiveness to parameters setting in genetic algorithm is improved. According to the comparisons with other algorithms, OMP_PA could precisely extract the pulse components, and the interferential components are almost filtered. The experiments show that, OMP_PA could determine the fault location of bearings, and clearly displayed the vibration model. In conclusion, it provides a new way to the diagnosis for bearings.
\end{abstract}

Keywords orthogonal matching pursuit; fault diagnosis; pulse atom; multi-population genetic algorithm; atomic decomposition

\section{Introduction}

Rolling bearing, as an important mechanism, is widely used in rotating machinery, but is also easily damaged ${ }^{[1]}$. The most effective way to improve its reliability is the real time monitoring of bearing vibration signals, whose cores are signal processing and fault feature extraction. The vibration signal of rolling bearing with localized defect in early stage is extremely complex and non-station, and the fault features are very weak. Atomic decomposition with time-frequency atoms proposed by Mallat and Zhang ${ }^{[2]}$ and Chen et al. ${ }^{[3]}$ in recent years has provided a new approach to this problem. Liu et al. ${ }^{[4]}$ applied matching pursuit with Gabor atoms (MP_GA) 
to the detection of localized defect of rolling bearing. Yang et al. ${ }^{[5]}$ chose the Symlet- 8 wavelet dictionary, and employed basis pursuit (BP) to analyze the vibration signal of bearing.

When using atomic decomposition for diagnosis, the vital issue is selecting proper atom and excellent pursuit method. In recent years, the pulse atom based on bearing vibration model was introduced and successfully applied in diagnosis ${ }^{[6-7]}$. Because the pulse atom is induced from the vibration model of faulty bearings, it is suitable for the pulse components in the vibration signal. However, there still exist some shortcomings. The number of atoms in dictionary is very big for high resolution, so selecting the best matching atom is quite computationally demanding in one iteration. Thus, artificial intelligent algorithms ${ }^{[7-8]}$ such as genetic algorithm (GA), particle swarm algorithm, have been applied to improve computational speed. However, the search of the best suitable pulse atom is an NP-hard problem, and conventional optimization algorithm is hard to find the exact solutions. In order to enhance the efficiency, an improved multi-population GA was employed to find the best matching atom. Although matching pursuit (MP) is a good approach of atomic decomposition, the orthogonal matching pursuit (OMP) $)^{[9]}$ which evolves from MP and makes all the bases be orthogonal to each other frequently has better resolution than MP. Therefore, OMP was used in this paper to decompose vibration signals. The experiments verified that the proposed scheme performed better than MP_GA and BP with Symlet-8 wavelet packets (BP_S8), and it could accurately extract the pulse components and correctly determine the fault location.

\section{The Principle of OMP with Pulse Atoms (OMP_PA) Optimized by Multi-Population GA}

\subsection{Introduction of Atomic Decomposition}

The conventional atomic decomposition methods include MP, BP, method of frames $(\mathrm{MOF})^{[10]}$, best orthogonal basis ${ }^{[11]}$, etc. Among all of them, OMP is the most popular atomic decomposition method which is used in face recognition, image processing, and so on. The common used dictionaries are Gabor dictionary, wavelet or wavelet packet dictionary, adaptive learning dictionary $^{[12]}$, Fourier dictionary, Dirac dictionary, compound dictionary, and so on.

\subsection{Introduction of OMP}

OMP, which improved from MP, is also an iterative greedy algorithm. First, the atom which matches the signal most is chosen. After choosing the best suitable atom in one iteration, OMP would make all the bases be orthogonal to each other and project them on the original signal again, then the matching component is extracted from the signal. The residual signal is further decomposed in the same way until a desired approximation precision or a prespecified number of iterations is reached. Owing to its simple steps and adaptability to the local structure of signal, OMP is the most popular one among atomic decomposition approaches. Therefore, OMP is chosen to decompose signals in this paper.

Suppose $D=\left\{g_{\gamma}\right\}_{\gamma \in \Gamma}$ is the overcomplete dictionary, $g_{\gamma}$ is the atom defined by the parameter group $\gamma$, and it is normalized as $\left\|g_{\gamma}\right\|=1, \Gamma$ is a parameter collection of atoms, signal $s$ is to be analyzed. The procedure of OMP is as follows ${ }^{[9]}$ :

Step 1: Initialize the residual $\boldsymbol{R}^{1}=\boldsymbol{s}$, the index set $\boldsymbol{\psi}_{0}=\emptyset$, and the iteration counter $n=1$. 
Step 2: Find the index of the basis that satisfies:

$$
\left|\left\langle\boldsymbol{R}^{n}, \boldsymbol{g}_{\gamma}^{n}\right\rangle\right|=\sup _{\gamma \in \Gamma}\left|\left\langle\boldsymbol{R}^{n}, \boldsymbol{g}_{\gamma}^{n}\right\rangle\right|
$$

The artificial intelligent methods can be applied in this step for fast calculation.

Step 3: Augment the matrix of the chosen basis: $\boldsymbol{\psi}_{n}=\boldsymbol{\psi}_{n-1} \cup\left\{\boldsymbol{g}_{\gamma}^{n}\right\}$.

Step 4: According to the least square problem the coefficients are estimated: $\boldsymbol{a}_{n}=\left(\boldsymbol{\psi}_{n}^{\mathrm{T}} \boldsymbol{\psi}_{n}\right)^{-1}$. $\boldsymbol{\psi}_{n}^{\mathrm{T}} \boldsymbol{s}$, and the residual is $\boldsymbol{R}^{n}=\boldsymbol{s}-\boldsymbol{a}_{n} \boldsymbol{\psi}_{n}$.

Step 5: Augment $n=n+1$, and return to Step 2 if $n$ is smaller than the maximum iterations. Otherwise, output the estimated reconstructed signal $\boldsymbol{s}_{k}=\boldsymbol{a}_{n} \boldsymbol{\psi}_{n}$ and its residual $\boldsymbol{R}^{n}$.

Equation (1) shows that selecting the best matching atom using inner products results in large calculation, hence how to reduce the large calculation is a challenge.

\subsection{The Vibration Model of Faulty Bearing — Pulse Sequence Model}

For rolling bearing, the outer race is generally fixed or is relatively fixed; the inner race and the shaft rotate together. The vibration signal of normal bearing is dominated by low frequency components caused by shaft rotation, stiffness variation, load fluctuation, etc. When a localized defect is induced, repeated pulses will be generated due to the passing of the rolling elements over the defect. Because the pulse is a broadband energy, which often sets off several modes of high-frequency resonances of the bearing elements, the neighboring structure, sensor, and so on. When the shaft speed is a constant, the time interval of the pulses is also a constant, and the corresponding frequency is termed as "fault passing frequency". When the defect occurs on the outer ring, inner ring or ball, the corresponding fault passing frequency varies, which can be used to determine the location of the fault.

In fact, there must always be some slip because the contact angle of rolling element varies, which results in $1 \% \sim 2 \%$ errors $^{[1]}$ of fault passing frequency. Therefore, the pulse sequence is quasi-periodic, which is $T$ and the period $T$ can be expressed as follows:

$$
x(t)=\sum_{k=0}^{+\infty} A_{k} h[t-i T+\lambda]+n(t)
$$

where $A_{k}$ is the amplitude of the $i$ th pulse, $t$ is time, $h(t)$ is a pulse oscillation caused by the crash of localized defect, $\lambda$ is the error caused by slip, and $n(t)$ is a random noise.

The pulse resonance can be simplified as follows:

$$
h(t)=\mathrm{e}^{-P_{0} t} \sin \left(2 \pi f_{n} t+\phi\right)
$$

where $f_{n}$ is the resonance frequency, $P_{0}$ indicates the damping speed of the pulse.

Cui et al. ${ }^{[13]}$ reported an improved model showing that two pulse resonances will be generated when the rolling elements passing over the single defect, as depicted in Figure 1(a). Cui et al. thought the two pulse resonances had the same features but only differed in beginning time. However, the real fault shape is often irregular, as showed in Figure 1(b), and the vibration modes are not the same, so that the two pulse resonances are frequently not the same either. Especially when the inner race or the ball is defect, due to the change of load, the amplitude of 
pulses varies, in some cases just the big pulse resonance is distinct. Therefore, the pulses were searched one by one in this paper, unlike combining two pulses as an atom in Cui's scheme.

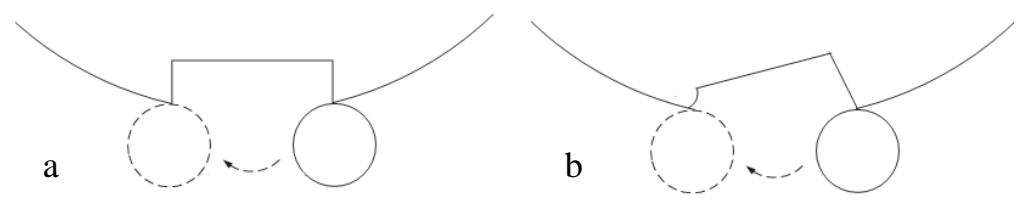

Figure 1 Physical model: (a) ideal model; (b) real model

\subsection{Pulse Dictionary}

\subsubsection{The Function of Pulse Atom}

From discussed above, one pulse resonance could be expressed as a pulse atom, which is shown as below:

$$
g_{\gamma}= \begin{cases}c \mathrm{e}^{-p(t-\tau)} \sin [2 \pi f(t-\tau)+\phi], & T_{s}>t \geq \tau \\ 0, & 0 \leq t<\tau\end{cases}
$$

where $g_{\gamma}$ is a normalized pulse atom indexed by the parameter group $\gamma, T_{s}$ is the duration of the signal $s ; p$ is the damping coefficient, the larger $p$ is, the faster the pulse decays; $\tau$ is time delay, namely the starting time of the pulse; $f$ is frequency. It represents the resonance frequency, and is often of high frequency for faulty bearings; $c$ is a normalization coefficient. The inner product $\left\langle s, g_{\gamma 0}\right\rangle$ denotes the pulse amplitude. In general, the more severity the fault is, the greater the amplitude is, and the pulse amplitude of the faulty bearing is usually larger than that of the normal one.

\subsubsection{The Discrete of the Pulse Parameters}

To improve the resolution of the decomposition, the density of the atoms in the dictionary is high, namely the discrete steps are small. The steps of the parameters of pulse atom are as follows:

1) The step of $p$ is 1 .

2) The step of $\tau$ is chosen as the resolution of the signal which is $1 / f_{s}$.

3) The step of $f$ is 1 .

4) The step of $\phi$ is $\pi / 64$.

According to the bearing geometry and signal character, the ranges of these atomic parameters are selected as follows:

1) Because the pulse decays fast, the parameter $p$ should be a large one. In this paper, it is chosen: $300 \leq p \leq 4000$.

2) $\tau$ must be smaller than the time duration of the vibration signal and above zero: $0<$ $\tau<T_{s}$, where $T_{s}$ is the time duration of the signal.

3) According to the Sampling Theorem, $f$ must satisfy the following conditions: $f<f_{s} / 2$, where $f_{s}$ is the sampling frequency of the signal. For the signal investigated in this paper, the majority energy of signals distributes below the frequency of $4000 \mathrm{~Hz}$, therefore, it is chosen: $0<f<4000 \mathrm{~Hz}$.

4) It is known that phase should satisfy: $0 \leq \phi \leq 2 \pi$. 


\subsection{Multi-Population GA}

Many kinds of optimized methods have been already applied to atomic decomposition for reducing the amount of calculation, such as GA, particle swarm algorithm, etc. GA has strong robustness and global search capability ${ }^{[14]}$, which is one of the most popular evolution algorithms of artificial intelligent methods. At the same time, GA has some urgent problems, for instance, premature convergence, sensitive to the parameters of crossover probability $p_{c}$ and mutation probability $p_{m}$. In view of the imperfections of GA, improved multi-population $\mathrm{GA}^{[14-17]}$ is employed, which introduces the following strategies to GA:

1) Selecting different control parameters. The values of $p_{c}$ and $p_{m}$ determine the equalization of global and local search, $p_{c}$ decides the global search capability, while $p_{m}$ the local search capability. Many researchers suggest a bigger $p_{m}(0.7 \sim 0.9)$ and a smaller $p_{c}(0.001 \sim 0.05)$. However, there still exist many different selections of $p_{c}$ and $p_{m}$, and for different selections, the outcomes vary greatly. Multi-population GA sets different values of $p_{c}$ and $p_{m}$ for each population, makes them co-evolve, and hence better balances the global and local search.

2) Subpopulations are independent, they communicate through migration operators. Migration operators introduce each subpopulation's best individuals regularly (a certain evolution generations) to replace the worst ones of the next subpopulations. In this way, the subpopulations exchange information and co-evolve.

3) In the evolution process, through manual selection operator, the best individuals of all of the subpopulations in each generation will be saved in the elite subpopulation. Elite subpopulation is just as a criterion to end iteration, i.e. when the elite subpopulation remains constant for a certain generations, the algorithm stops. This criterion fully exploits the accumulation of knowledge in the evolution process, and is more reasonable compared to GA, which takes a maximum number of iterations as the ending criterion. Also it overcomes the defect that the maximum number of iterations is difficult to be chosen correctly.

After these improvements, multi-population GA realizes a cooperative search between subpopulations, and enhances the equalization of global and local search. As a result, it tremendously reduces the bad influence of improper control parameters, and has a very impressive effect on the premature convergence problem.

\section{Experiments and Discussion}

\subsection{Comparison and Discussion Based on Simulated Experiments}

In order to test the performance of MP_PA, one vibration signal generated by imitated single point defect was simulated. The sampling frequency is $12000 \mathrm{~Hz}$, with the length as 2048 points. The simulated signal $y(t)$ is of five pulses added with Gaussian noise with signal to noise ratio (SNR) of $3 \mathrm{~dB}$.

$$
\begin{aligned}
y^{\prime}(t)= & 2 \cdot g_{1}(1200,200,3400, \pi / 2)+2 \cdot g_{2}(1200,600,3400, \pi / 2)+2 \cdot g_{3}(1200,1000,3400, \pi / 2) \\
& +2 \cdot g_{4}(1200,1400,3400, \pi / 2)+2 \cdot g_{5}(1200,1800,3400, \pi / 2) \\
y(t)= & y^{\prime}(t)+\text { white noise }(S N R=3)
\end{aligned}
$$

where $g(p, \tau, f, \phi)$ represents a pulse atom. It should be noted that for a convenient representation here $\tau$ denotes the number of signal sample. For example, when $\tau=1000$, it means 
that the pulse occurs at the moment of $1000 / f_{s}=0.0833$ second. The simulated signals are displayed in Figure 2.


Figure 2 Simulated signal: (a) signal $y^{\prime}(t)$; (b) signal $y(t)$

\subsubsection{The Comparison and Discussion of Multi-Population GA and GA}

Set $p_{c}=0.7, p_{m}=0.05$ in GA, whereas $p_{c}$ of any subpopulation in multi-population GA was a random decimal between $0.7 \sim 0.9$, and $p_{m}$ was a random decimal between 0.001 0.05. The number of chromosomes in GA was the same as that of all the subpopulations in multipopulation GA, and other parameters were selected the same. All the Matlab program were run in a computer with a dual-core $2.2 \mathrm{GHz}$ CPU and DDR $6674 \mathrm{G}$ memory.

In the simulated experiment, multi-population GA and GA were both used to optimize OMP to decompose the signal $y(t)$, and then the SNR and mean square error (MSE) between the reconstructed signal and $y^{\prime}(t)$ was calculated. The results are showed in Figure 3 and Table 1. It should be noted that, when the amplitude of the reconstruction and that of original signal are inverse, at the same time the sum of their phases is about $2 \pi$, and obviously the $\phi$ is still right.

As showed in Figure 3 and Table 1, for multi-population GA, the accuracy ratios of $\tau, f$ and amplitude are all very high, only that of $p$ is slightly low. In contrast, the results of GA are inferior. The SNR and MSE of multi-population GA are one order of magnitude higher than those of GA. The residue by multi-population GA is little by contrary, there still exist pulse components in the residue for GA. The running time of them are almost the same. These results show that multi-population GA performs much better than GA for optimizing OMP with regard to the vibration signal of faulty bearing.
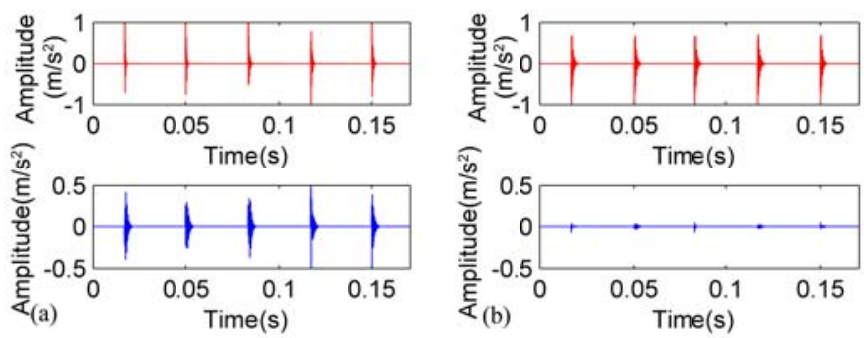

Figure 3 Reconstructions and residues of signal $y(t)$ optimized by GA and multi-population GA

(the numbers of atoms are both 5) 
Table 1 The decompositions optimized by GA and multi-population GA

\begin{tabular}{ccccccccc}
\hline Algorithm & Amplitude & $p$ & $\tau$ & $f$ & $\phi$ & SNR & MSE & Time \\
\hline \multirow{2}{*}{ GA } & 1.8983 & 1074 & 200 & 3335 & 1.252 & & & \\
& 1.6575 & 2953 & 601 & 3557 & 6.234 & 8.09 & 0.039 & $35 \mathrm{~s}$ \\
& -1.9419 & 2537 & 1001 & 3377 & 2.648 & & & \\
& 1.7693 & 2382 & 1401 & 3307 & 6.075 & & \\
Max error ratio & -1.9566 & 1892 & 1800 & 3527 & 4.850 & & \\
\hline & -1.9948 & 1180 & 200 & 3403 & 4.781 & & \\
Multi-population GA & -2.0484 & 1064 & 600 & 3404 & 4.710 & $\mathbf{2 2 . 3 0}$ & $\mathbf{0 . 0 0 8}$ \\
& 1.9604 & 1224 & 999 & 3440 & 1.634 & & \\
\hline Max error ratio & $\mathbf{3 . 6 \%}$ & $\mathbf{9 . 2 \%}$ & $\mathbf{0 . 1 \%}$ & $\mathbf{1 . 2 \%}$ & $\mathbf{0}$ & & \\
\hline
\end{tabular}

\subsubsection{The Comparison and Discussion of OMP_PA, MP_GA and BP_S8}

When using atomic decomposition to analyze vibration signals, the vital issues are choosing proper pursuit method and atom. In order to verify the performance of OMP_PA, different atomic decomposition algorithms and different dictionaries should be chosen for comparison, MP_GA and BP_S8 have been successfully used in bearing diagnosis ${ }^{[4-5,8,18]}$, which were chosen in this paper for a comparison. And the MP_GA was also optimized by GA.

The TFD by OMP_PA was calculated based on the parameters of the pulse atom. For a pulse atom $g(p, \tau, f, \phi)$, its frequency was $f$, and its amplitude decreased from the moment $\tau$ to the end of the signal. Therefore, let $G_{\gamma}=\left|c \mathrm{e}^{-p(t-\tau)}\right|$ as amplitude, we plotted it in TFD at the frequency of $f$ from the beginning moment of $\tau$ to the end of the atom. At last, the TFD of all the pulse atoms were summed up. Gabor atom and Symlet- 8 atom were discrete of power of 2 of scale as the same way as references ${ }^{[4-5,8,18]}$.

The results are shown in Figure 4 and Table 2, and the result of OMP_PA is shown in Figure 3(b), Table 1. Though MP_GA and BP_S8 could reconstruct the main pulse components of the origin, compared with OMP_PA, their sparsity is insufficient. And their reconstructions are contaminated with interference. In Figure 5, for MP_GA and BP_S8, the amplitude, energy and frequency of each pulse are different from others. And the distributions of the frequency are in a wide area, this will result in energy leakage. What's more, there are some noises. Although OMP_PA uses the least atoms, it could filter noises efficiently, and the pulses are correctly recovered. MP_GA used many atoms so that it wasted the most time. Whereas BP_S8 used all the atoms to find the sparse representation, thus it wasted the same time no matter how atoms in reconstruction.

The reason why OMP_PA performs so excellently is that pulse atom matches the pulses well, and OMP_PA has high resolution due to small step of parameters. By contrast, Gabor atom and Symlet-8 atom cannot match the pulses so well and they have to compensate the 
error with more atoms.
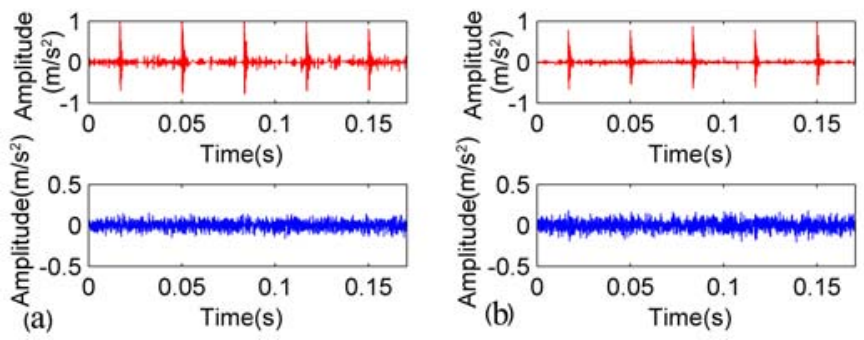

Figure 4 Reconstructions and residuals of signal $y(t)$ by different methods (the numbers of atoms are both 150): (a) MP_GA; (b) BP_S8
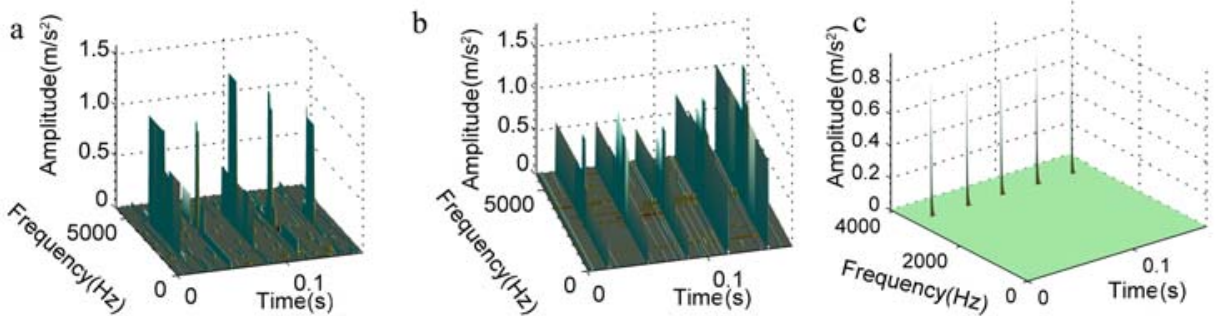

Figure 5 The TFDs of the reconstructions by different methods (the numbers of atoms are both 150): (a) MP_GA; (b) BP_S8; (c) OMP_PA

Table 2 The results by MP_GA, BP_S8 and OMP_PA

\begin{tabular}{ccccc}
\hline Method & Number of atoms & SNR & MSE & Time \\
\hline \multirow{2}{*}{ MP_GA } & 150 & 7.49 & 0.0515 & $70 \mathrm{~s}$ \\
& 300 & 11.01 & 0.0334 & $138 \mathrm{~s}$ \\
\hline \multirow{2}{*}{ BP_S8 } & 150 & 10.52 & 0.0294 & $83 \mathrm{~s}$ \\
& 300 & 8.86 & 0.0357 & $83 \mathrm{~s}$ \\
\hline OMP_PA & $\mathbf{5}$ & $\mathbf{2 2 . 3 0}$ & $\mathbf{0 . 0 0 7 6}$ & $\mathbf{3 1 ~ \mathbf { ~ }}$ \\
\hline
\end{tabular}

\subsection{Validation by Experiments}

The experiment data used in this paper were obtained from the Case Western Reserve University (CWRU) website. The signals were collected from SKF 6205 bearings which were seeded with faults using electro-discharge machining of outer race fault, inner race fault and ball fault. The sampling frequency is $12000 \mathrm{~Hz}$, the shaft rotation speed is about 1730 rotations one minute, namely $28.83 \mathrm{~Hz}$, and the bearing geometry is shown in Table 3 .

Table 3 Fault information

\begin{tabular}{cccc}
\hline Failure type & Fault passing frequency $(\mathrm{Hz})$ & Fault passing period $(\mathrm{ms})$ & Fault severity $(\mathrm{mm})$ \\
\hline Normal & - & - & - \\
Outer race & 103.5 & 9.7 & 0.178 \\
Inner race & 156.1 & 6.4 & 0.533 \\
\hline
\end{tabular}




\subsubsection{The Diagnosis and Analysis of the Bearing with Outer Race Fault}

The signal analyzed for outer race fault is measured under load of $3 \mathrm{hp}(1 \mathrm{hp}=746 \mathrm{~W})$ at 6:00 direction. As Figure 6 shows, OMP_PA has successfully extracted the pulse components with little noise, only using 18 atoms.
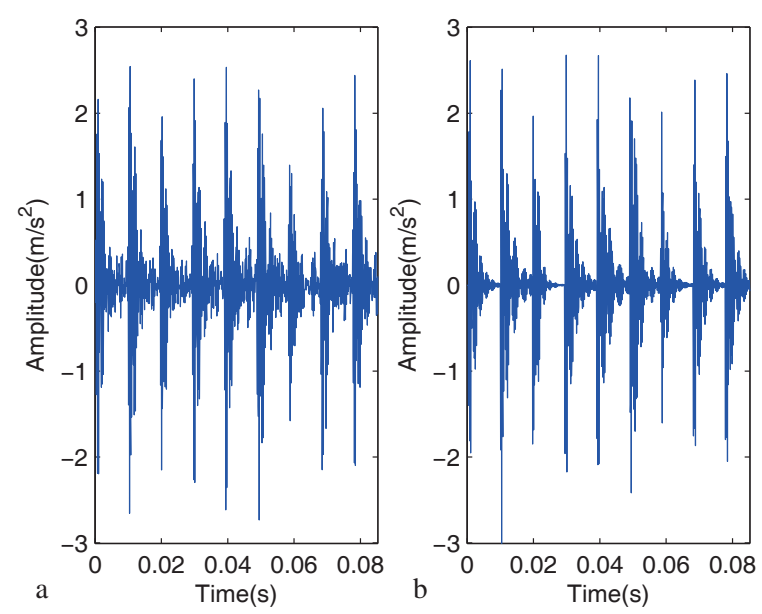

Figure 6 The original signal of the bearing with outer race fault and its reconstruction by OMP_PA

Figure 7 is the TFD and its corresponding pulse sequence by OMP_PA. It can be seen two pulse resonance sequences in TFD at frequencies of $3400 \mathrm{~Hz}$ and $2840 \mathrm{~Hz}$, and they have the same period. The pulse resonance sequence at $3400 \mathrm{~Hz}$ is a little earlier than that at 2840 $\mathrm{Hz}$, and it has higher energy. This demonstrates that the passing over the single defect point generated two pulse resonances, the early one is at $3400 \mathrm{~Hz}$, and the later one is at $2840 \mathrm{~Hz}$. The period of the pulse sequence is more clearly displayed in Figure 7(b), which is $9.7 \mathrm{~ms}$, corresponding to the outer fault passing frequency of $102.8 \mathrm{~Hz}$. These demonstrate that not only the fault location is determined, but also the vibration model could be obtained, which is convenient to further analyze for fault diagnosis.
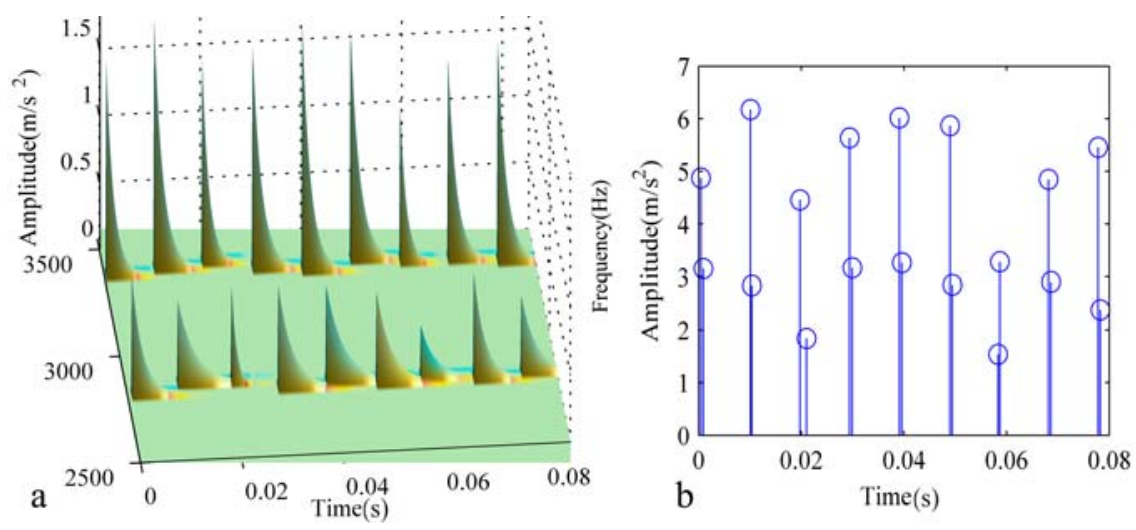

Figure 7 The TFD of the signal of the bearing with outer race fault and its pulse sequence 


\subsubsection{The Diagnosis and Analysis of the Bearing with Inner Race Fault}

As Figure 8 shows, the reconstruction by OMP_PA reserves the main pulses. From the reconstruction, it presents that the pulses of inner race fault vary in amplitude, which corresponds to the vibration model discussed above.
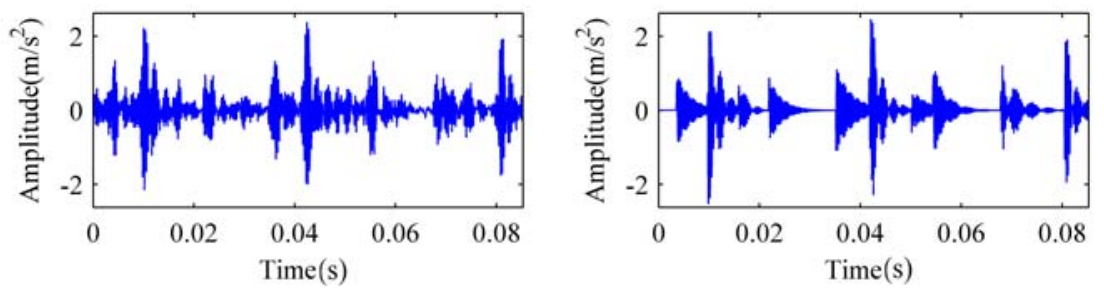

Figure 8 The original signal of the bearing with inner race fault and its reconstruction by OMP_PA

According to Figure 9, there is a pulse resonance sequence at frequency of $2800 \mathrm{~Hz}$, and the amplitude varies. A little later the high pulses, some pulses occurs at about $3300 \mathrm{~Hz}$, corresponding to the two pulse model. But when the energy of pulse is small, the pulses in company are buried in noise were not recovered, which is also accord to the mechanism of bearing. From Figure 9(b), the period is approximate $6.4 \mathrm{~ms}$, which corresponds to the inner race passing fault frequency of $155.3 \mathrm{~Hz}$. In addition, the resonance frequency distribution of inner race fault differs from that of the outer race fault, which can be used as a basis to distinguish the status of a bearing.
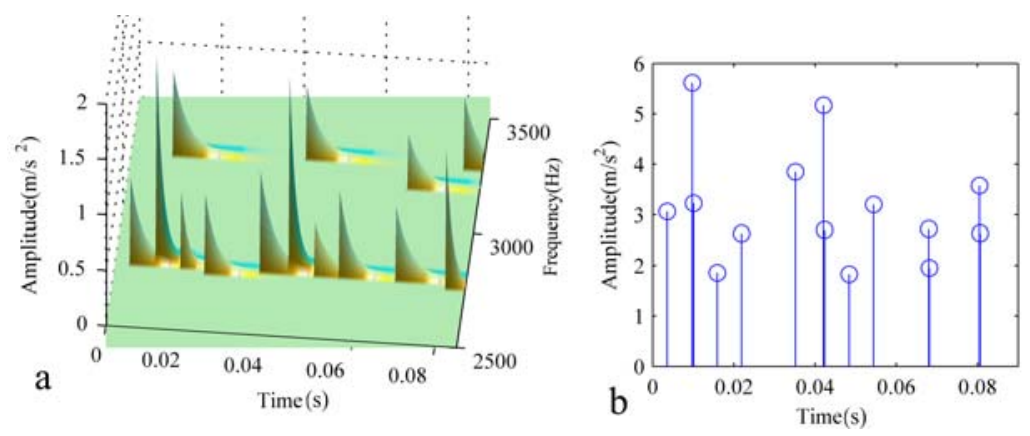

Figure 9 The TFD of the signal of the bearing with inner race fault and its pulse sequence

\subsubsection{The Distinguishing and Analysis of Normal Bearing}

In the rotation of normal bearing, owing to shaft rotation, stiffness variation, fluctuation, and so on, the signal will be dominated by low frequency vibration and noises, as shown in Figure 1(a). Its vibration waveform is often disorganized with small amplitude, and sometimes it contains weak pulses with the frequency proportional to shaft speed.

As illustrated in Figure 10(b), the reconstruction by OMP_PA has a distortion compared to the origin, due to that the pulse atom is insensibility to normal vibration, but only matches the pulse components. Whereas the bad reconstruction is also a particular basis of OMP_PA to confirm the bearing is normal. In the TFD by OMP_PA as Figure 11, there is a weak pulse resonance sequence whose period is about $34.8 \mathrm{~ms}(28.7 \mathrm{~Hz})$ at around $2000 \mathrm{~Hz}$, corresponding 
to the shaft rotation speed. In Figure 11(b) the dark pulses indicates the pulse resonances in Figure 11(a) around $2000 \mathrm{~Hz}$. There are also other phenomena to prove that the bearing is normal. The majority of pulse amplitudes are below 0.05 which is much less than those of faulty bearings. In addition, the frequencies of pulse atoms distribute in low frequency.
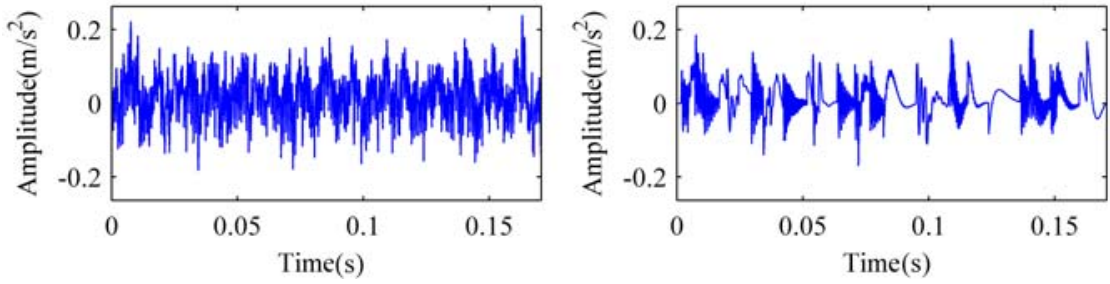

Figure 10 The original signal of the normal bearing and its reconstruction by OMP_PA
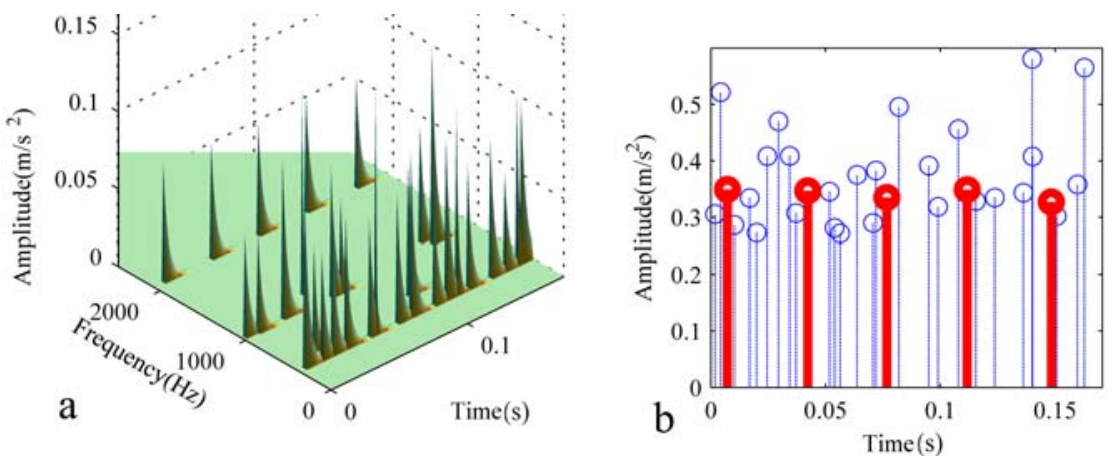

Figure 11 The TFD of the signal of the normal bearing and its pulse sequence

\section{Conclusion}

This paper presented a new technology for bearing fault diagnosis utilizing OMP with pulse atoms, which was based on the vibration model of faulty bearing. Because the proposed algorithm was very computationally demanding, multi-population GA was used to optimize OMP to improve efficiency verified by experiments, and multi-population GA got more accurate solutions than GA. OMP_PA was further investigated in simulations and experiments, and compared with MP_GA and BP_S8. The results showed that, OMP_PA represented the simulated signal more sparsely and accurately. From the vibration signals of bearings under different conditions, OMP_PA determined the status of the bearing clearly, and demonstrated the vibration model which was consistent with the model proposed in this paper for further investigation. In conclusion, the proposed scheme provided a new approach for bearing diagnosis.

\section{References}

[1] Randall R B, Antoni J. Rolling element bearing diagnostics - A tutorial. Mechanical Systems and Signal Processing, 2011, 25(2): 485-520.

[2] Mallat S G, Zhang Z. Matching pursuits with time-frequency dictionaries. IEEE Transactions on Signal Processing, 1993, 41(12): 3397-3415.

[3] Chen S S, Donoho D L, Saunders M A. Atomic decomposition by basis pursuit. SIAM Journal on Scientific Computing, 1998, 20(1): 33-61. 
[4] Liu B, Ling S, Gribonval R. Bearing failure detection using matching pursuit. NDT \& E International, 2002, 35(4): 255-262.

[5] Yang H, Mathew J, Ma L. Fault diagnosis of rolling element bearings using basis pursuit. Mechanical Systems and Signal Processing, 2005, 19(2): 341-356.

[6] Qin Q, Jiang Z N, Feng K, et al. A novel scheme for fault detection of reciprocating compressor valves based on basis pursuit, wave matching and support vector machine. Measurement, 2012, 45(5): 897-908.

[7] Wang X, Zhu H, Wang D, et al. The diagnosis of rolling bearing based on the parameters of pulse atoms and degree of cyclostationarity. Journal of Vibroengineering, 2013, 15(3): 1560-1575.

[8] Peng F, Yu D, Luo J. Sparse signal decomposition method based on multi-scale chirplet and its application to the fault diagnosis of gearboxes. Mechanical Systems and Signal Processing, 2011, 25(2): 549-557.

[9] Tropp J A, Gilbert A C. Signal recovery from random measurements via orthogonal matching pursuit. IEEE Transactions on Information Theory, 2007, 53(12): 4655-4666.

[10] Daubechies I. Time-frequency localization operators: A geometric phase space approach. IEEE Transactions on Information Theory, 1988, 34(4): 605-612.

[11] Coifman R R, Wickerhauser M V. Entropy-based algorithms for best basis selection. IEEE Transactions on Information Theory, 1992, 38(2): 713-718.

[12] Liu H, Liu C, Huang Y. Adaptive feature extraction using sparse coding for machinery fault diagnosis. Mechanical Systems and Signal Processing, 2011, 25(2): 558-574.

[13] Cui L, Wang J, Lee S. Matching pursuit of an adaptive impulse dictionary for bearing fault diagnosis. Journal of Sound and Vibration, 2014, 33(10): 2840-2862.

[14] Zhong W, Liu J, Xue M, et al. A multiagent genetic algorithm for global numerical optimization. IEEE Transactions on Systems, Man, and Cybernetics, Part B: Cybernetics, 2004, 34(2): 1128-1141.

[15] Li Y, Zhang S, Zeng X. Research of multi-population agent genetic algorithm for feature selection. Expert Systems with Applications, 2009, 36(9): 11570-11581.

[16] Zhou Q, Luo W. A novel multi-population genetic algorithm for multiple-choice multidimensional knapsack problems. Advances in Computation and Intelligence. Springer, Berlin Heidelberg, 2010: 148-157.

[17] Zhu H, Jiao L, Pan J. Multi-population genetic algorithm for feature selection. Advances in Natural Computation. Springer, Berlin Heidelberg, 2006: 480-487.

[18] Feng Z, Chu F. Application of atomic decomposition to gear damage detection. Journal of Sound and Vibration, 2007, 302(1): 138-151. 